\title{
ALUVIÓN EN EL CHICÓN
}

\section{Representaciones sociales del un desastre glaciar en Urubamba}

Liset Verónica Tecsi Conza ${ }^{1}$, Jhojan Rubén Tupa Ramos ${ }^{2}$.

\section{RESUMEN}

En esta investigación se indagó sobre algunas representaciones sociales contemporáneas de los pobladores de la Cuenca Chicón, quienes han construido un conocimiento colectivo en torno al desencadenamiento del aluvión del 2010, y antecedentes similares en 1679 y 1942. La perspectiva teórica de las representaciones sociales ha permitido conocer la teoría popular sobre las adjudicaciones políticas y naturales del evento así como sus efectos y acciones post desastre.

PALABRAS CLAVE: Representaciones Sociales, Cuenca Chicón, desastres, aluvión.
ABSTRACT
This research investigated some contemporary social representations of the inhabitants of the Cuenca Chicón, who have built a collective knowledge about the triggering of the flood of 2010, and similar backgrounds in 1679 and 1942. The theoretical perspective of social representations has allowed to known the popular theory about the political and

1 Licenciado en Antropología por la Escuela Profesional de Antropología de la Universidad Nacional de San Antonio Abad del Cusco.E-mail: vero.auc@gmail.com

2 Licenciado en Antropología por la Escuela Profesional de Antropología de la Universidad Nacional de San Antonio Abad del Cusco. 
natural adjudications of the event as well as its effects and post disaster actions.

KEYWORDS: Social Representations, Cuenca Chicón, disasters, alluvium.

E $\mathrm{n}$ las últimas décadas del siglo XX, el cambio climático ha ocasionado cambios significativos a nivel mundial, su impacto negativo sobre los glaciares tropicales se ha denominado retroceso glaciar. Los Andes peruanos contienen el 71\% de los glaciares tropicales de Sudamérica que están distribuidos en 19 cordilleras nevadas ${ }^{3}$. El derretimiento de masa glaciar está asociado a eventos catastróficos denominados aluviones.

La Cordillera del Urubamba posee alrededor de 117 glaciares de los cuales se han perdido el $61.69 \%$ de cobertura glaciar entre 1970 y $2009^{4}$, esta cifra implica la pérdida del recurso hídrico y mayor afectación a la población aledaña. El retroceso glaciar del nevado Chicón $(5,530$ m.s.n.m.) ubicado al norte de la ciudad de Urubamba ha ocasionado eventos aluviales en los años 1679, 1942 y 2010 a lo largo de la Cuenca Chicón ${ }^{5}$. Por ello, nuestro propósito es entender cómo los pobladores de dicha cuenca elaboran un conocimiento social del fenómeno a través de sus testimonios y experiencias vividas.

\section{MARCO TEORICO Y METODOS DE LA INVESTIGACION}

En primer lugar, incidimos en los aportes teóricos de las Representaciones sociales y el enfoque social de los desastres.

Jodelet (2000:10)) discípula de Moscovici, concluye que "las Representaciones Sociales conciernen al conocimiento del sentido común, que se pone disposición en la experiencia cotidiana". Por ello, la Representación Social existe en la cotidianeidad, construye significaciones simbólicas y también las reproduce. Entonces si conciernen al sentido común podemos incluir en ellas, las maneras de percibir, los modos de construir teorías de la vida cotidiana, además nos permiten conocer la realidad e interpretarla, se expresan en la interacción entre los individuos y el mundo a través de sus discursos, además están ligadas al lenguaje y a las prácticas sociales, puesto que a su vez funcionan como un tipo de lenguaje por la función que poseen y el marco que les rodea. Bajo este enfoque se han recolectado las percepciones, creencias o conocimiento popular de hombres y mujeres que han experimentado y han sido afectados por el aluvión de 1942 y 2010.

Pedro Ferradas (2015) detalla una serie de enfoques en torno al desastre, primero fue concebido como castigo de los dioses, luego como actuación maléfica de la naturaleza y posteriormente enfoques que consideran los factores naturales y la exposición de las personas y sus bienes, otorgándole un papel relevante a la conducta de las personas, organizaciones e instituciones. La importancia de los últimos enfoques radica en la relevancia de las capacidades locales para la respuesta, la percepción de las personas afectadas como sujetos de derecho y por tanto de participación en la emergencia.

Romero y Maskrey (1993:6) intentan diferenciar los términos, fenómeno natural y desastre natural, los cuales no son iguales, ni se superponen:

“...un terremoto que ocurre en un desierto deshabitado no puede considerarse como desastre, aunque sea de fuerte intensidad. Un terremoto solo causa desastre cuando afecta directamente o indirectamente al hombre y sus actividades en un lugar y tiempo determinado. En general, se considera como desastre natural a la coincidencia entre un fenómeno natural peligroso (inundación, terremoto, sequia, ciclón, etc.) y

3 Autoridad Nacional del Agua. (2014). Inventario de Glaciares y Lagunas. Unidad de Glaciología y Recursos Hidricos. Lima.

4 Según señala la Autoridad Nacional del Agua. (2014). Inventario de Glaciares del Perú. Huaraz: Unidad de Glaciología y Recursos Hidricos.

5 El uso del término cuenca Chicón proviene del mapa de peligros de la Municipalidad Provincial de Urubamba, en donde se le denomina Cuenca Hidrológica Quebrada Pumahuanca y Chicón. (Extraído: http://sial.muniurubamba.gob.pe/index.php? accion $=$ verElemento\&idElementoInformacion $=862 \&$ verPor $=$ \&idTipoElemento $=4$ \&idTipoFuente $=$ ) 
determinadas condiciones vulnerables. Existe el riesgo de que ocurra un desastre cuando uno o más peligros naturales se manifiestan en un contexto vulnerable." (Maskrey, 1989:19)

En otras palabras, se puede decir que hay un alto riesgo de desastre si uno o más fenómenos naturales peligrosos ocurrieran en situaciones vulnerables.

Bajo las premisas anteriores, ahora se puede entender la responsabilidad que tienen los hombres en la producción de los desastres "naturales", sabiendo que los fenómenos naturales ningún daño causarían si hubiéramos sido capaces de entender cómo funciona la naturaleza y de crear nuestro hábitat acorde con este conocimiento. Por ello, ponemos entre comillas naturales por la evidente construcción social del desastre, en definitiva el hombre podría reducir riesgos y vulnerabilidades a través del conocimiento y las prácticas sociales cotidianas.

\section{Métodos de la investigación}

La presente investigación es de tipo descriptivo. Las unidades de observación son la población damnificada de la Cuenca Chicón, condiciones de vida de la población, las representaciones sociales (creencias, opiniones y valoraciones) y las acciones sociales de la población frente al desastre glaciar. Técnicas de investigación: revisión de artículos periodísticos, observación del recorrido del aluvión, conversaciones casuales y entrevistas semiestructuradas a informantes clave.

\section{RESULTADOS}

\section{Cuenca Chicon}

Las aguas provenientes del Chicón atraviesan las comunidades de San Isidro de Chicón, Yanaconas, Chichubamba y Qatan Pino distribuidas en la parte alta, media y baja de la cuenca. Asentados más de 2000 pobladores, cuya actividad económica es en torno al cultivo de hortalizas, rosas, crianza de animales menores y trabajos relacionados al sector turismo. Cada comunidad se organiza en torno a la asamblea comunal, sus respectivas juntas directivas y comisiones específicas. A nivel intercomunal, la Junta de Regantes de la Cuenca Chicón representa la máxima autoridad, por ende cumple un rol integrador y activo. La Municipalidad Provincial de Urubamba realiza obras de saneamiento básico, infraestructura educativa y vial en favor de las comunidades y se cuenta con la presencia de organismos no gubernamentales.

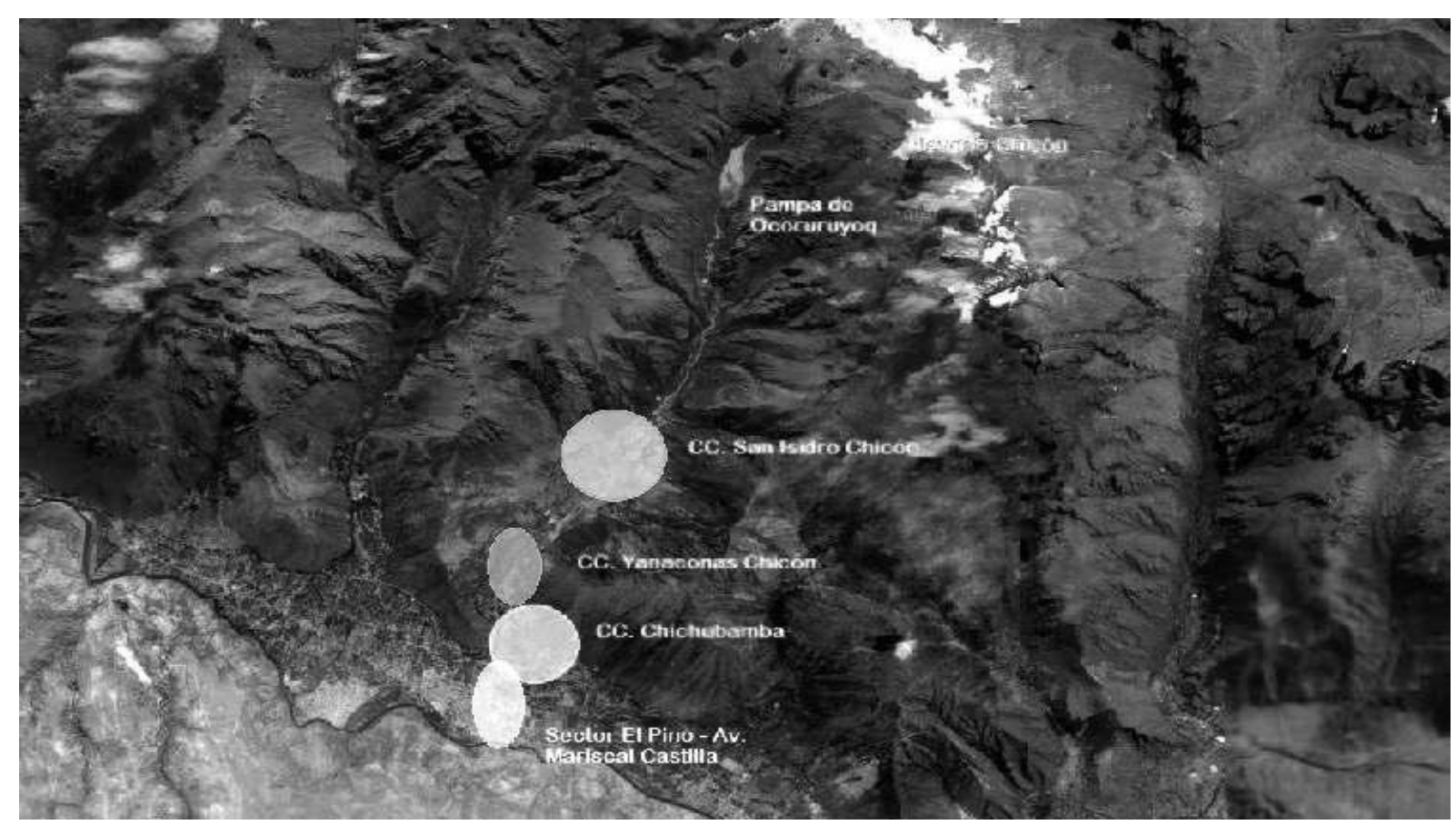

Fuente: Elaboración propia sobre imagen satelitalde Google Earth. 
Representaciones sociales del desastre glaciar

\section{El Apu Chicón se aleja}

Saliendo del Cusco, después de una hora de viaje, se avizoran una serie de cumbres nevadas, el Chicón y el Pumahuanca, advierten el ingreso a la benemérita ciudad de Urubamba. Llegamos a la av. Mariscal Castilla, amplia, ruidosa y abundante de negocios comerciales, cuyo recorrido nos lleva al cementerio local, punto de partida hacia el nevado Chicón, el rio del mismo nombre atraviesa el barrio Qatan Pino y las comunidades de Chichubamba, Yanaconas y San Isidro de Chicón.

Un recibimiento cálido, propio de la zona de valle, nos obliga a guarecernos en la chichería de don Eduardo para refrescarnos un poco y preguntarle qué está pasando con el Apu Chicón, y nos contesta que:

"antes todo era blanco, cerradito, he estado viendo desde mi niñez y ese nevado era hasta abajo blanco, y ahora ya no, ni la cuarta parte ya no hay nada [...] mi papá siempre hablaba "ritti urmayamushan", está cayendo la nevada, casi interdiario caía, que tremendas rocas habrá caído. (E.M. 63)

Con nostalgia del paisaje, el informante compara el nevado de "antes" y el actual, la imagen de hace unos treinta o cuarenta años no es la misma. Efectivamente, el nevado se aleja, se retira, retrocede rápidamente y genera preocupación por el desabastecimiento futuro del recurso hídrico.

\section{Un aluvión en 1942}

Estudios geológicos y registros históricos aseveran que en 1942, el Chicón arrasó con viviendas, animales, cultivos y además cobró vidas humanas, prueba de ello, inmensas rocas forman parte de los paisajes comunales. La catástrofe, como es citada por diarios de la época, fue de gran intensidad y conmoción nacional.

Este testimonio brinda una explicación mágico - religiosa del desastre: “...mi abuelita me conto que ese año estaba justo haciendo una fiesta y de un momento a otro ha llegado (refiriéndose al aluvión), los que han visto dicen que ha podido venir un hombrecito con un poncho rojo y todos decían que habrá venido pues el diablo." (N.Q., 60)

Un personaje desconocido con poncho rojo visita una fiesta familiar e inmediatamente se genera el desastre. Esta versión la relacionamos con la crónica de Guamán Poma, quien describe cómo un pequeño pueblo arequipeño es azotado por la furia de un hombre de pobres vestiduras que es rechazado en una fiesta del pueblo por su aspecto, su ira provoca prácticamente la desaparición del pueblo. Estos hombrecillos extraños son personificaciones del mal, que a modo de venganza destruyen ciudades y generan caos. En esta época aún no se habían divulgado los estudios geológicos y de riesgo local, por lo tanto, la población creaba personajes y situaciones de manera colectiva.

\section{Desastre glaciar del 2010}

Para muchos cusqueños, el año 2010 es sinónimo de lluvias, inundaciones, y desastres en varios distritos. En febrero de este año se desbordó el rio Vilcanota y afectó a gran parte del valle sagrado de los Incas, y meses después otro incidente en Urubamba, volvió a alarmar a dicha población.

El macizo Chicón, un domingo 17 de octubre a horas de la noche, con sus aguas, lodo, y todo a su paso sorprende a las comunidades y barrios de la Cuenca:

"justamente ese día domingo era un campeonato, a las once de la noche que ha pasado por aquí, donde nosotros estábamos así, no, yo también estaba descansando, entonces me llamaron que hay huayco, una familia que vive más arriba nos ha alertado, entonces hemos ido y tenía un olor pero muy diferente..." (W.M., 38)

Como lo describen los damnificados, el aluvión de octubre de 2010 fue una tragedia, 
recuerdan inclusive el ruido de las piedras, el olor parecido a pólvora, los gritos de alarma, los llantos desesperados, las reacciones abruptas al huir del siniestro, la impotencia de perder sus bienes. Las sensasiones y acciones ante el desastre fueron relatadas al detalle, pues para el colectivo, ha sido un hecho significativo.

\section{Efectos del desastre}

Según los registros de evaluación de daños de la Oficina de Defensa Civil de la Municipalidad provincial de Urubamba, se describe el suceso de la siguiente manera:

“...desprendimiento de un bloque de hielo del Nevado Chicón, cayendo hacia la laguna Pukaqocha, el mismo que provoco el desembalse ocasionando un aluvión de gran magnitud, el mismo que a su paso afecto los Centros Poblados de San Isidro Chicón, Yanaconas Chicón, Sector Chichubamba y la Av. Mariscal Castilla con las calles transversales."

La población afectada según este registro es de 17,787, la fuente de información proviene directamente del Comité Provincial de Defensa Civil Urubamba, incidiendo además en 1200 damnificados.

\section{Daños sobre viviendas, infraestructura vial y saneamiento}

La mayor afectación se dio en los sectores cercanos al cauce del río Chicón y la parte baja de la cuenca, debido al desastre, algunas viviendas se destruyeron y sus ocupantes tuvieron que abandonar sus propiedades y alquilarse habitaciones más seguras, otros decidieron trasladarse al distrito y a la ciudad del Cusco.

La entrevistada lamenta la destrucción de su vivienda, hecho que la obligó a vivir en alquiler.

"Yo tenía mi casita y al lado mi tiendita todo eso se cayó se ha derrumbado hasta ahora sigue así ahora estoy viviendo en alquiler, ha afectado más el sector de mi casa como allá arriba hay un puente se había trancado un árbol y se ha ido por el otro lado recto se ha venido, mi casita como era la primera con fuerza ha llegado como ha venido con tremendas piedras lo habian ahuecado..." (B.C., 53 CC Yanaconas)

A su vez, los entrevistados nos relatan sobre los daños en sus terrenos y las pérdidas que sufrieron en sus cultivos:

"Todito nos ha arrasado todito ese maíz era mar todito de mi hermano, alli adelantecito nomas, ahora se ha perdido maíz esa fecha era flores, se ha tapado todo el maizal a malogrado esa fecha" (N.H., 55 CC Chichubamba)

Nos relatan sobre las pérdidas de sus animales menores, de cómo ellos veían que el lodo llevaba a sus animalitos, cuan tristes se sentían al ver que no podían hacer nada frente al aluvión:

"De mi corral se lo habia llevado el chancho, las gallinas, cuyes. Todo se lo ha llevado de la cocina todo lo habrá enterrado...” (B.C., 53 CC Yanaconas)

El desastre afecto la vía carrozable en los sectores de Chichubamba, Yanaconas hasta llegar a San Isidro Chicón, dicha vía se encontraba lleno de piedras y lodo, por ello, el acceso a las comunidades se hizo complicado, ya que los trabajos de limpieza tardaron muchos días. Por otro lado, los puentes peatonales para vehículos fueron destruidos en diferentes sectores, a su vez el servicio de agua y desagüe fue suspendido por varios días debido a que en muchos sectores se encontraban dañadas las tuberías.

Acerca de las causas del desastre glaciar del 2010

La población ha elaborado dos versiones acerca de las causas del desastre, por un lado, que a consecuencia del retroceso glaciar, enormes trozos de hielo han formado una laguna y el desborde de ésta ha generado el aluvión. Por otro lado, los pobladores ponen en duda la causa natural y culpabilizan a una ex autoridad de la provincia, que habría mandado a dinamitar la zona con la finalidad de extraer minerales. 
La entrevistada afirma que "nosotros" los hombres provocamos el calentamiento global, este testimonio da a conocer la preocupación y responsabilidad de que los desastres son producto de la influencia del hombre:

"Eso decían desde muy antes se está desglaciando los nevados hay desglaciamiento del nevado será también pues por el calentamiento global que estamos provocando nosotros se ha desbordado, pero si desde muy antes siempre estaba en peligro el chicón siempre estaba." (A.P., 45 años. MPU)

De las dos versiones, la segunda y más difundida, alude a un personaje de la política local, ex-alcalde ing. Benicio Ríos, quien junto a algunos pobladores habría provocado una explosión en la zona, luego involucran a éste burgomaestre con la empresa JERGO $^{6}$ para extraer mineral del nevado, dichos intereses privados no han sido aceptados por la población local, produciéndose protestas y desacuerdos en los años posteriores.

"Hay dos versiones, para mi tengo bien claro, yo he subido hasta arriba al mismo lugar donde qué ha pasado, para mi ha sido provocado es como una dinamita hubiese explosionado. [...] es provocado para mí, según mi versión, los demás no sé porque no notificaron, no han visto nada, han llevado geólogos han venido como se llama pero no han dicho nada que ha sido provocado. Ha sido gente de peso pesado por eso para que no haya investigación, está metido una persona de aqui que conoce el lugar para que este de guía, no.” (N.Q., 60 CC Yanaconas)

Los rumores crecieron en la población por haber hallado piedras esparcidas que al parecer son resultado de un explosivo, y por ello la formación de una nueva laguna llamada Rit'iqocha. Los comentarios de los pobladores señalan que personas ajenas a las comunidades habían avisado sobre el incidente y que uno de ellos, era un curandero, quien habría realizado una ofrenda al nevado, enviado por el ex alcalde: “...habia un hombre que había bajado de arriba tenía su poncho y su chullo y habian unos caballeros que estaban subiendo de Urubamba [...] los señores que escucharon esto eran de Chichubamba ellos estaban subiendo de Urubamba hacia el cementerio, esas dos personas se habian encontrado con el señor que estaba bajando de aquí el Hampiq o qeswahuatoq el que pone pago y les habia dicho: "cuidado que vayan arriba porque ahorita va a venir huayco de arriba porque he puesto pago porque Benicio Rios me ha hecho poner pago porque van a dinamitar ya va llegar el huayco les estaba diciendo..." (B.C., 53 CC Yanaconas)

En estos relatos, nuevamente aparece la figura de un hombre de poncho rojo, un desconocido "avisa" sobre la ocurrencia del desastre, entre rumores se dice que pocos lo han visto pero han hablado con él, y se hace llamar curandero.

Después del aluvión, en el 2012 la gestión provincial de Urubamba ejecutó un proyecto de gestión de riesgos para resarcir los daños ocasionados por el fenómeno. La ejecución de actividades del proyecto estuvo a cargo de la empresa contratista Jergo. Dicen los pobladores, que "los Jergo llevaban tubos y cemento arriba, qué quieren hacer en nuestro nevado, seguro quieren llevarse nuestra agua, nuestros recursos..." entre dudas y descontento con las actividades de la empresa, los pobladores interpretan el desfogue de la laguna Rittiqocha (intervención técnica para reducir riesgos futuros) como un atentado a sus recursos naturales, se producen desacuerdos entre los representantes comunales y la empresa, incluso se paraliza el proyecto.

\section{DISCUSIÓN Y CONCLUSIONES}

\section{Discusión}

Carey (2014) analiza las respuestas ante el cambio climático y las consiguientes catástrofes glaciares desde 1941 hasta el

6 Empresa que fue contratada por la Municipalidad Provincial de Urubamba, cuya obra es del tipo de gestión de riesgos y emergencias. Denominada: Instalación del servicio de protección en la Cuenca del Río Chicón, en el Distrito de Urubamba, Provincia de Urubamba - Cusco (2012) y su presupuesto fue de S/. 14'155,494.00 


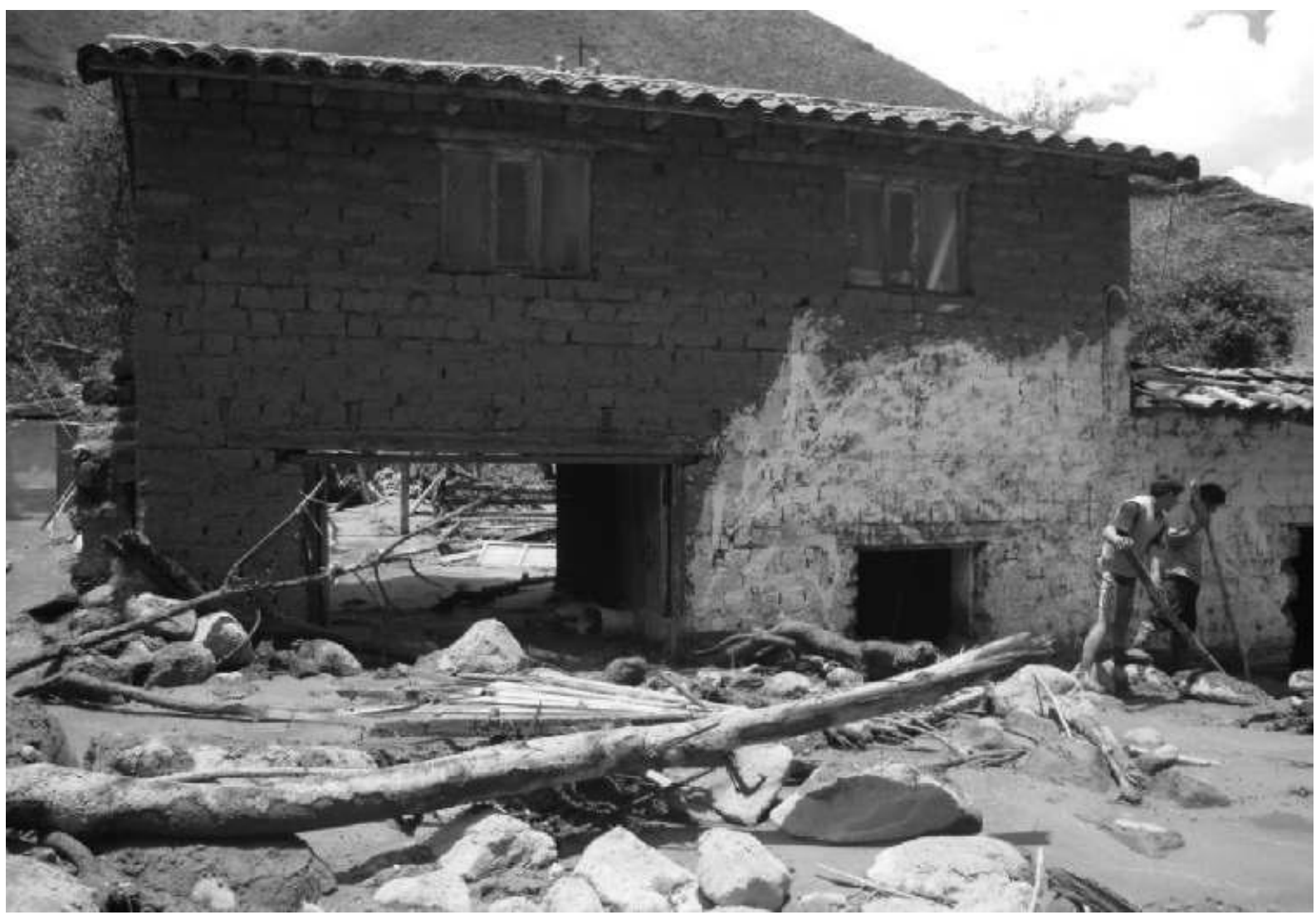

Daños causados en la vivienda de Nicolasa Quispe sector de Yanacona. Fuente Municipalidad de Urubamba.

presente. Su aporte a la historia ambiental resalta las hazañas de los científicos peruanos para advertir los riesgos del retroceso glaciar. Sin embargo, el conocimiento científico no es la única explicación sobre el origen de los desastres.

De manera similar, en la cuenca Chicón, se han realizado estudios geológicos $\mathrm{y}$ científicos que la sitúan como zona de peligro aluvionico, por su registro histórico posición geográfica. Sin embargo, los pobladores han elaborado sus propias explicaciones o teoría popular sobre el origen del aluvión, incluso rechazan las actividades de un proyecto de gestión de riesgos porque la versión de "la mano negra" o intereses de extracción minera es otra verdad que defienden.

En adelante, si se plantean medidas de apoyo y prevención de riesgos no será suficiente el conocimiento científico, sino su articulación con los conocimientos populares, y en este punto radica la importancia de conocer cómo se han construido socialmente estas explicaciones o significaciones.

Después de un sorpresivo desastre, qué acciones realizan los actores locales, cómo se activa el capital familiar, cómo se recuperan los afectados. Los pobladores de la Cuenca Chicón realizaron acciones de movilización a zonas seguras, pese a que muchos se vieron invadidos por el miedo y la desesperación, asimismo acciones de defensa tumbando árboles y asegurando sus viviendas. Entre las organizaciones locales resalta el papel de la Iglesia Evangélica, sus miembros fueron recordados por reparto de agua y alimentos. La participación de instituciones públicas y privadas como Instituto de Defensa Civil, el Gobierno Regional del Cusco, la Municipalidad Provincial de Urubamba se dio a través de la dotación de carpas, frazadas, herramientas, víveres, etc., y ONGs como PREDES realizaron visitas de expedición y acciones de señalización. Si bien realizaron acciones de apoyo que fueron reconocidas por la población rural, también hubo quejas 
sobre la ineficiente distribución de dicho apoyo.

\section{Conclusiones}

Al apoyarnos en el enfoque de las Representaciones Sociales de los pobladores de la Cuenca Chicón, hemos puesto énfasis en la teoría popular que "ellos" han elaborado sobre el origen del desastre. El aluvión 1942 se ha construido por la memoria colectiva como un hecho de gran intensidad cuyo origen se debe a la presencia de un personaje maligno. Y en el 2010, dos versiones explican las causas del desastre glaciar. La pérdida de masa glaciar y la intervención política de intereses extractivos. Este desastre afectó las viviendas, cultivos, infraestructura vial, servicios básicos, además de las condiciones de salud y educación de la población que presenta condiciones de vulnerabilidad altas.

Pese a las dificultades, se activó enormemente el capital social, la cooperación de los familiares y amigos, como la intervención de las instituciones públicas y privadas contribuyeron a la recuperación post - desastre.

En este escenario de riesgos climáticos es crucial articular esfuerzos tanto científicos como políticos para modificar hábitos $\mathrm{o}$ impulsar reformas que contribuyan a la defensa de los recursos naturales y la conciencia ambiental sobre la reducción de glaciares. El hombre andino, que vive en y con los Apus tutelares cumple un rol esencial en la protección de sus recursos y en la adaptación a nuevos escenarios.

\section{BIBLIOGRAFÍA}

Altamirano Vega, \& Altamirano Bonett. (2010). Geología, Geografia, Historia y Mitologia del Valle Sagrado de los Incas. Cusco.

Araya Umaña, S. (2002). Las Representaciones Sociales: Ejes Teoricos para su discusión. San Jose, Costa Rica: Facultad Latinoamericana de Ciencias Sociales FLACSO.

Autoridad Nacional del Agua . (2014). Inventario de Glaciares y Lagunas. Unidad de Glaciología y Recursos Hidricos. Lima: Autoridad Nacional del Agua.
Calvo Rossano (1994). Del folclore a la antropología del terremoto: el Señor de los Milagros (Lima) y el Señor de los Temblores (Qosqo). En Folclore Americano 57.

Cardona A., O. D. (1993). Evaluación de la Amenaza, la vulnerabilidad y el riesgo. En A. MASKREY, Los Desastres nos son Naturales (págs. 45 - 63). Red de Estudios Sociales en prevención de Desasatres en America Latina.

Carey, M. (2014). Glaciares, cambio climatico y desastres naturales. Ciencia y Sociedad en el Peru. Lima: IFEA - IEP.

Carlotto Caillaux, V. (2005). Mapa de Peligros de la Ciudad de Urubamba. Informe Final Proyecto INDECI-PNUD PER/02/051 Ciudades Sostenibles.

Carlotto, V. (2010). Evaluación Geologica y geodinámica en la quebrada Chicón: Aluvión del 17 de octubre que afectó Urubamba Cusco. Lima: INGEMMET.

Concha Tupayachi, S. (s.f.). Urubamba Provincia Arqueologica del Perù.

El Comercio, C. (28 de ENERO de 1942). Urubamba la perla del Vilcanota ha sido arrasado por una abalancha de agua y lodo, págs. 1-2.

El Comercio, C. (29 Jueves de Enero de 1942). Catastrofe del Vilcanota.

El Comercio, C. (31 de Enero de Enero de 1942). Auxilio a los Damnificados de la catastrofe de Urubamba. pág. 2.

ERFCC, C. (2012). Estrategia Regional Frente al Cambio Climatico. Cusco: Danny's Graff E.I.R.L.

Ferradas, P. (2015). La Memoria es tambien Porvenir. Historia mundial de los desastres. Lima: Soluciones Practicas.

Guaman Poma de Ayala, F. (s.f.). Nueva Coronica y Buen Gobierno.

Jodelet, D., \& Guerrero Tapia, A. (2000). Develando la Cultura. Estudios en Representaciones Sociales. Mexico, D.F: Universidad Nacional Autonoma de Mexico.

Maskrey, A. (1989). El manejo Popular de los Desastres Naturales. Estudios de Vulnerabilidad y Mitigación. Lima: Tenologia Intermedia ITDG.

Maskrey, A. (1993). Los Desastres no son Naturales. La Red. Red de Estudios Sociales en Prevención de Desastres en America Latina .

Maskrey, A., \& Romero, G. (1993). Como entender los Desastres Naturales. En A. MASKREY, Los Desastres no son Naturales (pág. 134). La Red. Red de Estudios Sociales en Prevención de Desastres en America Latina.

Moscovici, S. (1979). El Psicoanálisis, su imagen y su público. Buenos Aires: Editorial Huemul.

Municipalidad Provincial de Urubamba. (2007). Diagnostico Situacional y Plan de Desarrollo Estrategico Concertado. Distrito de Urubamba al 2020. Urubamba.

Romero Zeballos, G. (2010). Prevención. Boletin Informativo. Año 03 - Boletin 22 - Edicion Especial Cusco. Cusco: PREDES. Centro de Estudios y Prevención de Desastres.

Toscana Aparicio, A., \& VALDEZ PEREZ, V. (2014). Representaciones sociales del desastre de 1940 en Santa Cruz Pueblo Nuevo, Estado de México. Investigaciones Geográficas, Boletín del Instituto de Geografia, UNAM, 88-101.

Valladares Quijano, M. (2011). Yungay en la memoria . Lima: Pakarina.

Zans Candia, L. (2007). Urubamba, Benemérita Ciudad y Provincia Arqueológica del Peru. Cusco: JL. editores . 
